\title{
BMJ Randomised controlled trial of weekly Open chloroquine to re-establish normal erythron iron flux and haemoglobin recovery in postmalarial anaemia
}

\author{
Sharon E Cox,${ }^{1,2}$ Chidi V Nweneka, ${ }^{1,3}$ Conor P Doherty, ${ }^{4}$ Anthony J Fulford,,${ }^{1,3}$ \\ Sophie E Moore, ${ }^{1,3}$ Andrew M Prentice ${ }^{1,3}$
}

To cite: Cox SE,

Nweneka CV, Doherty CP, et al. Randomised controlled trial of weekly chloroquine to re-establish normal erythron iron flux and haemoglobin recovery in postmalarial anaemia. BMJ Open 2013;3 e002666. doi:10.1136/ bmjopen-2013-002666

- Prepublication history and additional material for this paper is available online. To view these files please visit the journal online (http://dx.doi.org/10.1136/ bmjopen-2013-002666).

CVN and SEC contributed equally.

Received 31 January 2013 Revised 11 April 2013 Accepted 30 May 2013

This final article is available for use under the terms of the Creative Commons Attribution Non-Commercial 3.0 Licence; see http://bmjopen.bmj.com

For numbered affiliations see end of article.

\section{Correspondence to}

Professor Andrew M Prentice; Andrew.Prentice@Ishtm.ac.uk

\section{ABSTRACT}

Objective: To determine if low-dose weekly chloroquine (CQ) therapy improves recovery from malaria-associated anaemia.

Design: Proof of concept randomised clinical trial. Setting: West Kiang District, Lower River Region, The Gambia.

Participants: Children resident in participating communities, aged 12-72 months, with uncomplicated malaria identified using active case detection over two consecutive malaria transmission seasons.

Interventions: In 2007, eligible children were randomised to chloroquine-sulfadoxine/pyrimethamine (CQ-SP) or co-artemether (ACT) antimalarial therapy, and after parasite clearance on day 3 were subsequently re-randomised (double-blind) to weekly low-dose CQ $(5 \mathrm{mg} / \mathrm{kg})$ or placebo. In 2008 , all eligible children were treated with $\mathrm{ACT}$ and subsequently randomised to $C Q$ or placebo.

Outcome measures: The primary outcome was a change in haemoglobin from baseline (day 3 of antimalarial treatment) to day 90 in the $C Q$ and placebo treatment arms. Secondary outcomes were changes in urinary neopterin as a marker of macrophage activation, markers of erythropoietic response and prevalence of submicroscopic parasitaemia. Change in haemoglobin in the placebo arm by initial antimalarial treatment was also assessed.

Results: In 2007, 101 children with uncomplicated malaria were randomised to antimalarial treatment with $C Q-S P$ or ACT and 65 were subsequently randomised to weekly $C Q$ or placebo. In 2008 , all children received ACT antimalarial treatment and 31 were subsequently randomised to receive weekly CQ or placebo. Follow-up to day 90 was $96 \%$. There was no effect of weekly $C Q$ vs placebo on change in haemoglobin at day $90(\mathrm{CQ}+10.04 \mathrm{~g} / \mathrm{L}$ $(95 \% \mathrm{Cl} 6.66$ to 13.42$)$ vs placebo $+7.61 \mathrm{~g} / \mathrm{L}(95 \% \mathrm{Cl}$ 2.88 to 12.35$)$ ). There was no effect on the secondary outcomes assessed, or effect of initial antimalarial therapy on haemoglobin recovery. Higher day 90 haemoglobin correlated independently with older age, not being stunted, higher haemoglobin at day 0 and adequate iron status at day 3 .

\section{ARTICLE SUMMARY}

Article focus

- To determine if 3 months of low-dose weekly chloroquine (CQ) $(5 \mathrm{mg} / \mathrm{kg})$ therapy after effective treatment of uncomplicated malaria can improve haemoglobin recovery after malaria-associated anaemia in rural African children.

- To test the hypothesis that chloroquine may enhance haematological recovery after a malaria episode through its anti-inflammatory and immuno-modulatory actions, antimacrophageal iron loading effect and antimalarial effect (despite widespread resistance).

Key messages

- Weekly low-dose CQ after effective antimalarial treatment was not effective in improving recovery from mild-to-moderate postmalarial anaemia.

- Weekly low-dose CQ at $5 \mathrm{mg} / \mathrm{kg}$ had no clear effects on erythropoietic responses, as determined by reticulocyte percentage, or immunemediated inflammation as assessed by urinary neopterin, a marker of macrophage activation.

Conclusions: Weekly low-dose $C Q$ after effective antimalarial treatment is not effective in improving recovery from postmalarial anaemia.

Trial registration: The clinical trial registration number is NCT00473837 (ClinicalTrials.gov).

\section{INTRODUCTION}

The precise contribution of malaria to anaemia is difficult to calculate mainly because many anaemia-causing conditions coexist in children living in malaria-endemic countries. ${ }^{1}$ Murphy and Breman ${ }^{2}$ estimate the annual incidence of severe malarial anaemia (SMA) to be 1.42-5.66 million, 


\section{ARTICLE SUMMARY}

Strengths and limitations of this study

- The strengths of this study are its randomised design and double blinding of the allocation to weekly $C Q$ or placebo and that few subjects were lost to follow-up. The continuous follow-up and active case detection for all children ensured that malarial infections were detected early and within similar time frames, plus resurgent or new clinical infections were excluded from the analysis of haemoglobin change. A strict malaria case definition was adhered to and the measurement of submicroscopic parasitaemia over time allowed the effects of incomplete treatment to be modelled.

- A limitation of the study was the need to recruit participants over two malaria transmission seasons and the significantly reduced malaria transmission rate in 2008 and thus reduced recruitment, which also appeared to affect the severity of malaria in terms of parasite densities at first detection and haemoglobin concentrations. However, Cls of the effect size in the final multivariable analysis do not include the clinically significant effect size included in the sample size calculation, and thus our observation is unlikely to be due to a type II error as a result of lack of power to detect a true effect.

- It is possible that the relatively low dose of CQ we used was insufficient to mediate the effects that we were testing for and we were unable to conduct pilot studies of $C Q$ drug dosage and duration to determine the potential best treatment regime to test or of the timings of evaluation of possible mechanisms of effect.

- The change in design in 2008 to all children receiving ACT antimalarial treatment did not affect the analysis of the primary outcome. The change did, however, limit our ability to determine the effect of initial antimalaria therapy on haemoglobin response in the placebo group. An effect of antimalarial treatment $\mathrm{CQ}$ on $\mathrm{Hb}$ when in the context of adequate parasite clearance, as was observed, might have been expected to occur in the early period of recovery.

resulting in between 194000 and 974000 deaths in children younger than 5 years of age. SMA increases the risk of death in children with malaria, ${ }^{3}$ many of whom die shortly after admission before blood transfusion can be started ${ }^{45}$ and after discharge in the community. ${ }^{6}$ In addition, blood transfusion, the standard treatment for severe malarial anaemia exposes the child to the risk of transfusion-related infections like HIV and hepatitis. ${ }^{7}$ Among a nationally representative sample of children aged 1-5 years in the Gambia in 1999, 76\% had a haemoglobin $(\mathrm{Hb})$ less than $11 \mathrm{~g} / \mathrm{dL}$ and $15 \%$ had $\mathrm{Hb}$ less than $7 \mathrm{~g} / \mathrm{dL}^{8}{ }^{8}$ It is quite likely that most of the cases of moderate-to-severe anaemia were due in great part to malaria. ${ }^{9-11}$ A better understanding of the pathogenesis of malarial anaemia is required to improve its prevention and management.

Multiple immune and non-immune-mediated mechanisms cause anaemia during and after a malarial episode ${ }^{12}$ including the destruction of non-parasitised erythrocytes, which may account for more than $90 \%$ of erythrocyte loss. ${ }^{13}$ One important component is inflammation-induced iron delocalisation, which is characterised by sequestration of iron by the monocytemacrophage system and hepatocytes, resulting in a state of pseudo-iron deficiency, a state that reverses after the inflammation has subsided. ${ }^{12}$ The trans-membrane protein ferroportin is the exclusive route for cellular iron efflux and is responsible for releasing absorbed iron from enterocytes and sequestered iron from macrophages. ${ }^{12} \quad 15 \quad 16$ Hepcidin, the master regulator of iron metabolism which responds both to iron status and inflammation, ${ }^{15}$ blocks ferroportin. Clinically, malaria-induced inflammation has been shown to be associated with a hepcidin-mediated reduction in intestinal absorption of iron. ${ }^{17}$ Inflammatory cytokines, particularly interleukin (IL)-6 and IL-1, stimulate hepcidin release, ${ }^{18} 19$ which results both in the reduced iron absorption of dietary iron and impaired macrophageal release of iron, the latter effect being quantitatively much more significant as macrophageal iron recycling and transport to the erythron are 25-fold to 30-fold greater than the influx of dietary iron. In this way, hepcidin is the mediator of the anaemia of chronic disease. ${ }^{15}$ Tumour necrosis factor $\alpha$ could also play a role in ferroportin-mediated iron transfer independent of hepcidin. ${ }^{20}$ Rapid resolution of malaria-induced inflammation could therefore limit the degree and duration of malarial anaemia by reducing the duration of iron delocalisation.

Chloroquine is a cheap and safe antimalarial drug with proven anti-inflammatory properties; it has been shown to reduce iron delocalisation in vitro. ${ }^{21}{ }^{22}$ We hypothesised that chloroquine may enhance haematological recovery after a malarial episode through its antiinflammatory and immuno-modulatory actions, antimacrophageal iron loading effect and antimalarial effect (despite widespread resistance). We designed a proof of concept study to investigate the potential use of chloroquine in the management of mild postmalarial anaemia.

\section{MATERIALS AND METHODS}

\section{Study design and population}

This study was a population-based randomised controlled trial (RCT) in children aged 12-72 months that was conducted over two malarial seasons in 2007 and 2008 in West Kiang District, Lower River Region, The Gambia. The study was initially designed and conducted as a $2 \times 2$ randomised placebo-controlled trial in 2007 but had to be changed, due to changes in the national malaria treatment guidelines, to a two-arm RCT during the second malaria transmission season in 2008. In the $2 \times 2$ phase of the study, children with malaria were initially openly randomised to receive a therapeutic dose regime of chloroquine plus sulfadoxine/pyrimethamine (CQ-SP) or co-artemether (ACT) (day 0). After treatment, on day 3 , children were subsequently randomised (double blind) to receive weekly chloroquine or placebo until day 90 (figure 1). In the two-arm phase, all 


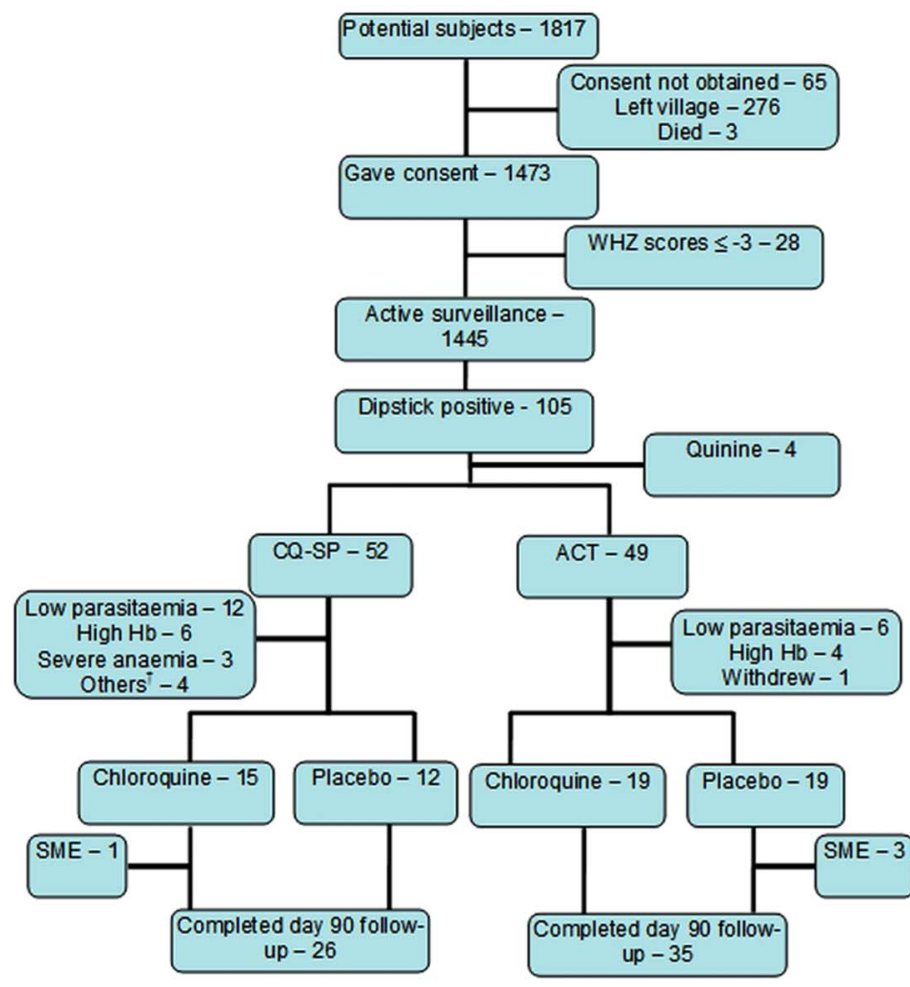

SNE = second malaria episode; $\dagger$ 'Others' include early treatment failure (ETF) -1 ; left village after day $3-1$; refused bleeding -1 ; and referred to the supplement centre -1

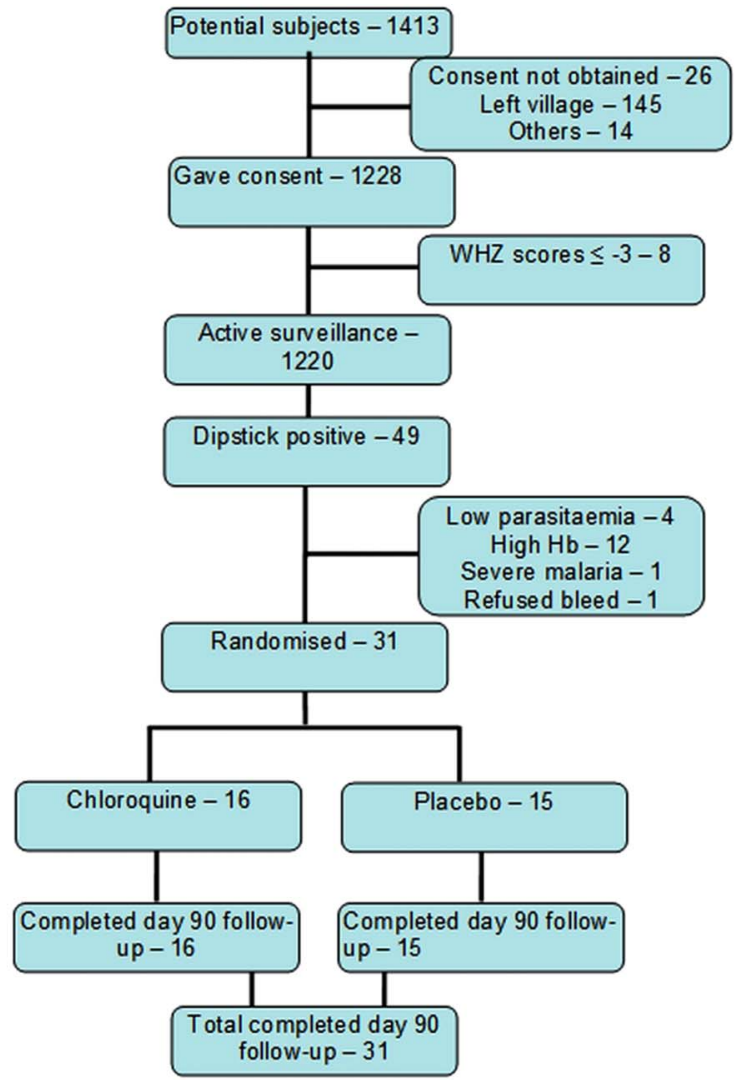

Figure 1 CONSORT flow diagram describing participants' movements in 2007 and 2008.

enrolled children with malaria were treated with ACT and then randomised and followed up as before (figure 1). A combination of active and passive case detection was used to identify children in participating communities with malaria using a standard case definition of clinical malaria (see below).

The climate of this district is typical of sub-Sahelian Africa with a long dry season lasting from November to June and a relatively short rainy season lasting from July to October. Malaria transmission is seasonal, hyperendemic and follows the rainy season, occurring between July and December. The prevalence of children experiencing one or more malarial episodes among a cohort of 1002 children aged between 1 and 6 years under active malaria surveillance during a population-based study in 2003 was 34\% (Doherty et al, unpublished data). During the study period, standard malaria control activities in the district consisted of insecticide-treated bednet distribution and case finding and treatment. In 2008, the standard first-line antimalarial treatment regimen for uncomplicated malaria in the Gambia was changed from CQ-SP to co-artemether.

\section{Inclusion and exclusion criteria}

Eligibility for malaria surveillance and subsequent possible enrolment into the RCT included all children in the participating communities, aged between 12 and
72 months, at the start of the surveillance period and who were not severely wasted, defined as weight-forheight $\mathrm{Z}$ scores less than -3SD.

Inclusion criteria for enrolment included: (1) uncomplicated malaria at day 0 , defined as a history of fever $48 \mathrm{~h}$ prior to presentation or a measured temperature of $37.5^{\circ} \mathrm{C}$ or more with peripheral parasitaemia (asexual forms of Plasmodium falciparum $\geq 500 / \mu \mathrm{L}$ by microscopy) on day 0 of the study and (2) for randomisation to the postmalarial intervention, children had to have an $\mathrm{Hb}$ between 69 and $110 \mathrm{~g} / \mathrm{L}$ on day 3 with no peripheral parasitaemia.

Children were excluded from the RCT if they were: (1) unable to take oral medications; (2) had features of severe malaria ${ }^{1}$; (3) had a known haemoglobinopathy; (4) were enrolled in another project; (5) had already received antimalarial drugs from other sources outside the project team or (6) were prescribed other drugs with potential antimalarial or antianaemic effects such as co-trimoxazole and haematinics.

Following randomisation to weekly $\mathrm{CQ}$ or placebo, children were withdrawn from the study if: (1) they developed a second malarial episode or had lifethreatening symptoms, more severe disease or serious adverse reactions; (2) left the study area for more than 14 days; (3) received antimalarials from other sources or (4) their $\mathrm{Hb}$ fell below $70 \mathrm{~g} / \mathrm{L}$. 


\section{Field and clinical procedures}

To effect the surveillance protocol, the study villages were divided into geographically adjacent clusters supervised by a resident study nurse who visited each village morning and evening and provided $24 \mathrm{~h}$ clinical coverage to all enrolled children in their cluster. The active surveillance consisted of twice weekly temperature monitoring of enrolled children by village assistants, while the passive surveillance consisted of mothers referring sick children to the visiting study nurse. Children with fever-reported or measured-were screened for malaria using a rapid diagnostic test (MRDT; Cortez diagnostics, Inc) after clinical evaluation. Children testing negative to MRDT were treated based on the likely diagnosis from the symptoms and signs. Children testing positive to MRDT were started on antimalarials immediately (either CQ-SP or ACT in 2007, and ACT in 2008 for 3 days) and directly observed by the study nurse and managed as appropriate. Finger prick blood samples (days 7, 15, 30 and 70) were collected in an EDTA microtainer while venous blood $(2 \mathrm{~mL}-$ days 0 and 90; and $5 \mathrm{~mL}$-days 3 and 45) samples were collected in an EDTA vacutainer and sent to the study laboratory on the same day. Comorbidities were treated as appropriate, avoiding co-trimoxazole and haematinics. For the subsequent visits after initial malaria diagnosis at day 0 , children were seen by the study nurse and blood samples and clinical data collected as per protocol. Only children who fulfilled the inclusion criteria for advancement to the weekly chloroquine/weekly placebo phase of the study were sampled after day 3 . These children were also followed up on days 15, 30, 45, 70 (except in 2008) and 90.

\section{Randomisation and blinding}

The randomisation to malaria treatment group in 2007 (CQ-SP or ACT) was open (non-blinded to trial staff, investigators or participants) and was carried out by sequential assignment as children were identified. The block randomisation to the postmalaria treatment of weekly CQ or placebo in both 2007 and 2008 was double blinded and was carried out in blocks of eight. The randomisation codes were generated by a staff member independent of the study team and held by the external trial monitor. Treatment codes were labelled A to $\mathrm{H}$ and placed in sequentially numbered, opaque sealed envelopes held by the study nurses. Allocation to the treatment was by matching the code in the envelope to a bottle of the intervention labelled with the same code and then labelled with the subject ID.

\section{Interventions}

Children openly randomised to malaria treatment at day 0 with CQ-SP received choroquine syrup (Chlormal, Medreich Sterilab Ltd, Avalahalli, Bangalore, India) consisting of $50 \mathrm{mg}$ chloroquine base per $5 \mathrm{~mL}$ as chloroquine phosphate at a dose of $10 \mathrm{mg} / \mathrm{kg}$ for 3 days (days 0-2). SP (Micro Laboratories, India) was co-administered as a $525 \mathrm{mg}$ tablet (500 $\mathrm{mg}$ sulfadoxine and $25 \mathrm{mg}$ pyrimethamine) on day 0 only at a dose of half tablet $/ 10 \mathrm{~kg}$. Children who were randomised to ACT treatment in 2007 and all children in 2008 received co-artemether (Coartem) tablets (Beijing Novartis Pharma Ltd, Beijing China for Novartis Pharma AG, Basle, Switzerland) as blister packets of six tablets per card with each tablet containing $20 \mathrm{mg}$ artemether and $120 \mathrm{mg}$ lumefantrine. The treatment dose comprised a stat dose on day 0 and then $8 \mathrm{~h}$ later, followed by twice daily doses for the next 2 days with doses depending on body weight ( 5 for $<15 \mathrm{~kg}, 1$ tablet/dose; 15 for $<25 \mathrm{~kg}$, 2 tablets/dose; 25 for $<35 \mathrm{~kg}, 3$ tablets per dose).

During the second blinded phase of the trial, children received either weekly CQ syrup (as above) but at a dose of $5 \mathrm{mg} / \mathrm{kg}$ or matching placebo syrup (prepared by the Royal Victoria Teaching Hospital pharmaceutical laboratory in collaboration with Atlantic Pharmaceuticals Limited, Latrikunda Sabiji, The Gambia) The syrups were in similar amber-coloured bottles with matching caps and labels. Doses were administered in the child's home under direct observation by the study nurses who waited for at least $10 \mathrm{~min}$ after administering the drugs to ensure that they were not vomited.

\section{Primary and secondary outcomes}

The primary outcome measure was $\mathrm{Hb}$ change from day 3 post-treatment of the malarial episode to day 90 in the weekly CQ and placebo arms.

The secondary outcomes were to compare between the weekly CQ and placebo arms: (1a) changes in erythropoietic response between days 3 and 30; and (1b) between days 3 and 90 ; (2) changes in urinary neopterin from days 3 to 30 as a marker of macrophage activation; (3) prevalence of submicroscopic malaria parasitaemia using PCR at each time point and (4) Hb change from days 3 to 90 in the two placebo arms to investigate the effects of initial malaria treatment therapy.

\section{Sample size}

During the initial study design, we used a working assumption of an SD for change in $\mathrm{Hb}$ of $15 \mathrm{~g} / \mathrm{L} .{ }^{23}$ With power $80 \%$ and significance level set at $5 \%$, we estimated the sample sizes required to detect varying effect sizes for the primary outcome. A sample size of 65 per arm was estimated to be able to detect an effect size of $7.5 \mathrm{~g} / \mathrm{L}$. This sample size was thought to be achievable and the effect size of likely public health significance.

\section{Laboratory procedures}

Table 1 describes the different laboratory analyses conducted. Zinc protoporphyrin (ZnPP) as a marker of iron status was measured on fresh whole blood using a fluorometer (AVIV Biomedicals, Lakewood, New Jersey, USA). Full blood count (FBC), haemoglobin and automated reticulocyte count were performed using a Cell Dyne 3700 (Abbott Laboratories, Abbott Park, Illinois, USA). Iron deficiency was defined using three 
Table 1 Laboratory investigations conducted

\begin{tabular}{|c|c|c|c|}
\hline Investigations & Specimen & Days collected* & Purpose \\
\hline Microscopy for malaria parasite & Blood & $0,3,7,15,30,45,70$ and 90 & To assess parasitaemia \\
\hline Erythrocyte zinc protoporphyrin & Blood & 0 and 3 & To assess iron status \\
\hline Full blood count & Blood & $0,3,7,15,30,45,70$ and 90 & To assess $\mathrm{Hb}$ change over time \\
\hline Automated reticulocyte percentage & Blood & $3,7,15,30,45,70$ and 90 & To monitor bone marrow response \\
\hline $\begin{array}{l}\text { Quantitative PCR for Plasmodium } \\
\text { falciparum parasites }\end{array}$ & Blood & $0,3,7,15,30,45,70$ and 90 & To assess submicroscopic parasitaemia \\
\hline Urinary neopterin concentration & Urine & 3,15 and 30 & $\begin{array}{l}\text { To assess macrophage activation as } \\
\text { measure of immune-mediated inflammation }\end{array}$ \\
\hline
\end{tabular}

parameters: mean cell volume (MCV), mean cell haemoglobin $(\mathrm{MCH})$ and $\mathrm{ZnPP}$. The cut-off values for the different parameters employed were: $\geq 61 \mu \mathrm{mol} / \mathrm{mol}$ of $\mathrm{Hb}$ for $\mathrm{ZnPP}^{24} \leq 73 \mathrm{fL}$ for $\mathrm{MCV}^{25}$ and $\leq 25 \mathrm{pg}$ for $\mathrm{MCH}^{25}$ Combining these parameters has been shown to increase their sensitivity and specificity to identify iron deficiency. ${ }^{26}$ Malarial parasites were identified in a thick peripheral blood smear stained with Giemsa overnight and read by a light microscope with $\times 100$ magnification in oil immersion. Parasite DNA extraction and subsequent quantitative PCR amplification were carried out using established protocols. ${ }^{27}$ In brief, DNA extraction was carried out using the QIamp DNA Mini Kit (Qiagen Ltd, UK) according to the manufacturer's instructions with minor modifications. Extracted DNA samples were stored at $-20^{\circ} \mathrm{C}$. Quantification of parasite density was performed with a TaqMan assay employing a fluorogenic probe to detect specific parasite products and using an Opticon 2 Real Time PCR machine and Opticon V.3.1 software (Bio-Rad Laboratories, Inc). Primers and probes were synthesised by Applied Biosystems, UK. Urinary neopterin levels were measured using an ELISA technique (BRAHMS Neopterin EIA, B.R.A.H.M.S, Germany). The stored urine was allowed to thaw at room temperature and then diluted $1: 100$, and the rest of the procedure was according to the manufacturer's instructions.

\section{Statistical analysis}

Delta haemoglobin summary statistics are presented as means and SDs for normally distributed continuous variables, and counts and percentages for discrete variables. Neopterin and parasite count were not normally distributed and were $\log$ transformed ( $\log _{\mathrm{e}}$ for neopterin and $\log _{10}$ for parasite count). The geometric means and 95\% CIs are presented for log-transformed variables. Two-sided Student $\mathrm{t}$ tests were used to compare results between two treatment arms.

Visual inspection of $\mathrm{Hb}$ change between baseline at day 3 and later time points by treatment arm suggested that, by day $30, \mathrm{Hb}$ change in both treatment arms had reached a plateau. To fully determine the effect of treatment group on $\mathrm{Hb}$ change, we used random effects models using the repeat measures at days 30 and 90 . Initial malaria treatment group (CQ-SP vs ACT), Hb at recruitment (day $0 \mathrm{Hb}$ ), baseline $\mathrm{Hb}$ (day 3), age in months, year of study, and the child's village were included in the models 'a priori' and hence adjusted for.

Linear regression was used to investigate further potential predictors of $\mathrm{Hb}$ change in the study population. First, simple linear regression was used to assess the association of each predictor variable with $\mathrm{Hb}$. Thereafter, all variables with a $\mathrm{p}$ value of 0.1 or less were included in a multiple linear regression to assess confounding. The final regression model consisted of only the variables that maintained a significant association with $\mathrm{Hb}$ in the multiple regression models. All analyses were carried out using STATA V.8 (StataCorp, College Station, Texas, USA).

\section{Ethical clearance}

The Scientific Coordinating Committee of the Medical Research Council (UK), The Gambia, and the MRC/ Gambia Government Joint Ethical Committee approved the study (SCC 1076). Written informed consent was obtained from parents/guardians of the children prior to enrolment. The clinical trial registration number is NCT00473837 (ClinicalTrials.gov).

\section{RESULTS}

\section{Characteristics of the study population under malaria} surveillance

Figure 1 describes the flow of participants through the study and the reasons for exclusions from the study. In 2007, 1445 of 1817 (80\%) potentially eligible children identified through the Keneba MRC DSS database from 14 villages were included in the malaria surveillance. In 2008, 1220 of the $1413(86 \%)$ identified children from five of the original villages were included in the malaria surveillance (smaller villages were excluded as they had contributed few cases in 2007). Table 2 describes the characteristics of the children placed under surveillance for malaria. The proportion of children with a positive malaria rapid test during surveillance (only first event per child counted) fell from $7.3 \%$ (105/1445) in 2007 
Table 2 Baseline characteristics of all participants under surveillance

\begin{tabular}{|c|c|c|c|}
\hline & $\begin{array}{l}2007 \\
N=1445\end{array}$ & $\begin{array}{l}2008 \\
N=1220\end{array}$ & $\begin{array}{l}\text { Combined } \\
\mathrm{N}=2665\end{array}$ \\
\hline Mean age in months (SD) & $42.2(17.2)$ & $40.7(17.7)$ & $41.5(17.5)$ \\
\hline Mean weight-for-height $Z$ scores (SD) & $-1.06(0.84)$ & $-1.02(0.85)$ & $-1.04(0.84)$ \\
\hline Mean weight-for-age $Z$ scores (SD) & $-1.48(0.97)$ & $-1.50(0.94)$ & $-1.49(0.95)$ \\
\hline Mean height-for-age Z scores (SD) & $-1.06(1.21)$ & $-1.17(1.13)$ & $-1.11(1.17)$ \\
\hline Mean body mass index $\left(\mathrm{kg} / \mathrm{m}^{2}\right)(\mathrm{SD})$ & $14.6(1.3)$ & $14.7(1.4)$ & $14.6(1.4)$ \\
\hline Prevalence of a malaria rapid test positive result during surveillance* & $105(7.3)$ & 49 (4.0) & $154(5.8)$ \\
\hline
\end{tabular}

to $4 \%(49 / 1220)$ in 2008 . In the first year of the study, $101(96 \%)$ of the children with a positive malaria rapid test were randomised to CQ-SP or ACT treatment, and $65(64 \%)$ subsequently randomised at day 3 to weekly chloroquine or placebo, with all children completing follow-up until day 30 and 33/34 completing follow-up until day 90 in the weekly CQ and 28/31 in the weekly placebo group. In the second year of the study, 31 of the $49(63 \%)$ cases with a first positive malaria rapid test were subsequently randomised to weekly $\mathrm{CQ}$ or placebo after treatment with ACT, all of whom completed follow-up until day 90 (16 CQ and 15 placebo). Thus, 96 children were included in the analysis of the primary and secondary outcomes, except for the analysis of effect of initial malaria treatment within the placebo arms which included 31 children.

Table 3 describes the characteristics of participants randomised to weekly chloroquine and weekly placebo for both years combined. Demographic and anthropometric characteristics were similar between the groups as was baseline $\mathrm{Hb}$ at day 3. The prevalence of iron deficiency measured at baseline was 43.1\% (28/65) in 2007 and $58.1 \%(18 / 31)$ in 2008 with a similar prevalence between the $\mathrm{CQ} /$ placebo groups within each year (data not shown) and in both years combined. However, parasite density was lower in the placebo group (table 3). No adverse events were reported in either treatment group.

\section{Effect of weekly CQ on haemoglobin change during} follow-up

To assess the effect of weekly chloroquine on $\mathrm{Hb}$ recovery after clearance of peripheral parasitaemia, we compared change in $\mathrm{Hb}$ from days 3 to 90 and from days 3 to 30 in the two arms. No significant differences in $\mathrm{Hb}$ change between the two groups were observed, either for the data for both study years or for each study year separately (both years combined: change in $\mathrm{Hb}$ at day 90, $\mathrm{CQ}+10.04 \mathrm{~g} / \mathrm{L}(95 \%$ CI 6.66 to 13.42$)$ vs placebo $+7.61 \mathrm{~g} / \mathrm{L}(95 \%$ CI 2.88 to 12.35$)$; figure 2$)$. Thus, the effect size of the CQ treatment was $2.43 \mathrm{~g} / \mathrm{L}(95 \%$ CI -3.38 to 8.24 ) compared to the $7.5 \mathrm{~g} / \mathrm{L}$ we included in the sample size calculation. However, at day 15 , children receiving weekly $\mathrm{CQ}$ had an increase of $3.5 \mathrm{~g} / \mathrm{L}(95 \%$ CI 0.5 to 6.4$)$ compared to $0.8 \mathrm{~g} / \mathrm{L}(95 \% \mathrm{CI}-2.3$ to 3.9$)$ in children in the placebo arm, but this difference did not reach statistical significance $(p=0.2)$. The lack of effect of weekly CQ remained when adjusting for factors determined 'a priori' ( $\mathrm{Hb}$ at recruitment (day 0 ), $\mathrm{Hb}$ at baseline (day 3), age (in months), year of study, initial treatment received (CQ-SP vs ACT) and village group) using a random effects repeated measures model including $\mathrm{Hb}$ measured at days 30 and 90 (coefficient $=1.43$ (95\% CI -1.70 to 4.57$)$. Furthermore, adding parasite density at recruitment, presence of submicroscopic parasitaemia at any time point during follow-up, and

Table 3 Baseline characteristics of children randomised to weekly chloroquine and weekly placebo in 2007 and 2008, pooled

\begin{tabular}{lcr}
\hline & $\begin{array}{l}\text { Chloroquine group } \\
\text { Parameter }\end{array}$ & $\begin{array}{c}\text { Placebo group } \\
\text { N=46 }\end{array}$ \\
\hline Male sex (\%) & $28(56.0)$ & $24(52.2)$ \\
Age in months, mean (SD) & $41.9(18.1)$ & $38.7(17.8)$ \\
Height-for-age Z scores, mean (SD) & $-1.19(0.91)$ & $-0.94(1.20)$ \\
Weight-for-age Z scores, mean (SD) & $-1.44(0.95)$ & $-1.35(0.87)$ \\
Weight-for-height Z scores, mean (SD) & $-0.94(0.90)$ & $-0.96(0.79)$ \\
BMl (kg/m ${ }^{2}$ ), mean (SD) & $14.7(1.4)$ & $14.7(1.3)$ \\
Parasite density at recruitment (day 0), geometric mean, (95\% Cl) & $47783(31617$ to 71120$)$ & $32496(21910$ to -47 507) \\
Hb (g/L) at recruitment (day 0), mean (SD) & $103.9(14.2)$ & $101.5(14.8)$ \\
Hb (g/L) at baseline (day 3), mean (SD) & $93.4(9.6)$ & $93.7(10.2)$ \\
Iron deficient ${ }^{*}$ at baseline (day 3) (\%) & $23(46)$ & $23(50)$ \\
\hline
\end{tabular}

*Iron deficiency was defined as zinc protoporphyrin $\geq 61 \mu \mathrm{mol} / \mathrm{mol}$ of $\mathrm{Hb}$, mean cell volume $\leq 73 \mathrm{fL}$ and mean cell haemoglobin $\leq 25 \mathrm{pg}$. 


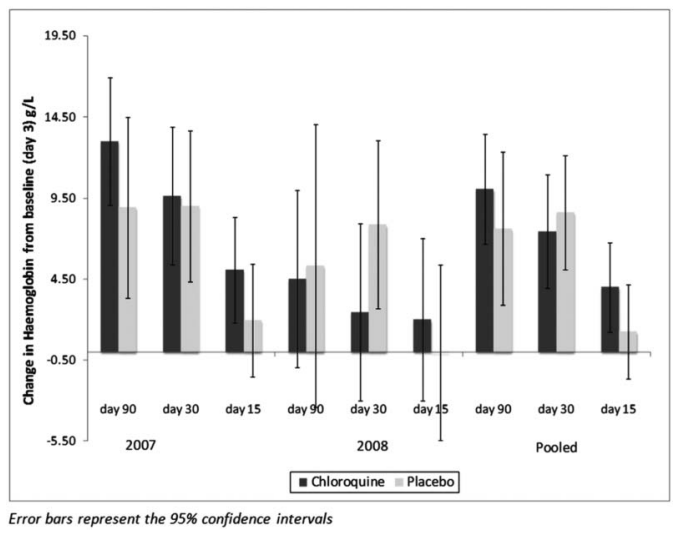

Figure 2 Mean change in haemoglobin concentration at days 30 and 90 from baseline at day 3 by treatment group for each year of study and for both years combined. Error bars represent the $95 \%$ Cls. Change in haemoglobin at day 3 is compared to haemoglobin concentration at presentation with malaria and initiation of antimalarial treatment. Thereafter, the comparison is with baseline levels at day 3 after completion of antimalarial treatment. Error bars represent the 95\% Cls.

presence of iron deficiency had no effect on the estimates of effect for $\mathrm{CQ}$ vs placebo treatment during follow-up (data not shown).

\section{Predictors of haemoglobin at day 90}

In the absence of an effect of weekly $\mathrm{CQ}$ on $\mathrm{Hb}$ change after successful malaria treatment, we assessed what other factors might determine final $\mathrm{Hb}$. Using simple linear regression, significant positive associations were observed for parasite density at day 0 , better nutritional status, age, $\mathrm{Hb}$ at day 0 at malaria diagnosis and day 3 of successful treatment. Presence of iron deficiency at baseline was associated with significantly lower final $\mathrm{Hb}$. There was no apparent effect of initial malaria treatment (CQ-SP vs ACT; table 4). In a final multiple regression model, $\mathrm{Hb}$ at day 0 was the strongest predictor of final
$\mathrm{Hb}$ and other independent predictors of final haemoglobin were presence of iron deficiency at day 3, degree of stunting (height-for-age $\mathrm{Z}$ score), and age (in months). Children with higher $\mathrm{Hb}$ at diagnosis and older children had greater final Hb. Children with iron deficiency at day 3 had significantly lower final $\mathrm{Hb}$ with a mean difference of $12.1 \mathrm{~g} / \mathrm{L}$ (table 5 ).

\section{Effect of initial antimalaria treatment (CQ-SP vs ACT) on haemoglobin change}

A secondary outcome of this study was $\mathrm{Hb}$ change from days 3 to 30 and day 90 in the placebo arm to determine whether initial antimalaria treatment (CQ-SP or ACT) treatment affected macrophage iron loading in acute clinical malaria and its subsequent release. As indicated in the analysis of $\mathrm{Hb}$ at day 90 , there was no significant effect of inital antimalarial treatment. Please see online supplementary figure $\mathrm{S} 1$ depicting $\mathrm{Hb}$ change during follow-up from days 0 to 90 in the placebo arm by initial antimalarial treatment received and the accompanying text.

Submicroscopic parasitaemia, inflammation, bone marrow response, haemoglobin change and treatment groups

Submicroscopic parasitaemia was commonest in the first 7 days of acute phase follow-up (days 3-15). By day 30, most were negative $(77 \%)$. The prevalence of submicroscopic parasitaemia at days 30 and 90 combined was $15.1 \%$ and there was no evidence of an effect of the postmalaria treatment group or of initial antimalarial treatment (figure 3 and see online supplementary figure S2). Most of the submicroscopic parasitaemia at these time points was of a very low level (1-2 parasites $/ \mu \mathrm{L})$. However, higher parasite density at diagnosis was associated with an increased likelihood of subsequent submicroscopic parasitaemia at days 30 or 90 with a one-log increase in parasite count increasing the odds of having submicroscopic parasitaemia by $1.6(\mathrm{p}=0.005)$. Although the presence/absence of submicroscopic parasitaemia

Table 4 Predictors of final haemoglobin at day 90 using univariable linear regression

\begin{tabular}{|c|c|c|}
\hline Parameter & Regression coefficient $(95 \% \mathrm{Cl})$ & p Value \\
\hline Parasite count at recruitment (day 0) & $3.5(1.1$ to 5.8$)$ & 0.004 \\
\hline Sex & $-0.4(-3.3$ to 2.5$)$ & 0.8 \\
\hline Iron deficiency at baseline (day 3) & $-12.1(-14.7$ to -9.5$)$ & $<0.001$ \\
\hline Height-for-age $Z$ scores $(H A Z)<-2$ & $3.2(1.8$ to 4.6$)$ & $<0.001$ \\
\hline Weight-for-age $Z$ scores (WAZ) $<-2$ & $2.6(1.0$ to 4.2$)$ & 0.001 \\
\hline Weight-for-height (WHZ) Z scores $<-2$ & $0.1(-1.6$ to 1.8$)$ & 0.9 \\
\hline Age in months & $0.4(0.3$ to 0.4$)$ & $<0.001$ \\
\hline Initial antimalaria treatment arm & $1.2(-2.1$ to 4.4$)$ & 0.5 \\
\hline CQ vs placebo randomisation group & $-0.5(-3.4$ to 2.5$)$ & 0.8 \\
\hline $\mathrm{Hb}(\mathrm{g} / \mathrm{L})$ at recruitment (day 0$)$ & $0.6(0.5$ to 0.6$)$ & $<0.001$ \\
\hline $\mathrm{Hb}(\mathrm{g} / \mathrm{L})$ at baseline (day 3) & 0.7 (0.6 to 0.8$)$ & $<0.001$ \\
\hline Village & $1.9(0.9$ to 3.0$)$ & $<0.001$ \\
\hline Presence of sub-microscopic parasitaemia at any time point during follow-up & $0.6(-7.1$ to 8.2$)$ & 0.9 \\
\hline Year of study & $-1.3(-4.4$ to 1.8$)$ & 0.4 \\
\hline
\end{tabular}


Table 5 Independent predictors of final haemoglobin (day 90) using multivariable linear regression

\begin{tabular}{llr}
\hline Parameter & Regression coefficient $(95 \% \mathbf{C l})$ & $\mathbf{p}$ Value \\
\hline Day 0 Hb & $0.44(0.36$ to 0.52$)$ & $<0.001$ \\
Presence of iron deficiency & $-3.0(-5.57$ to -0.43$)$ & 0.022 \\
Height-for-age Z score $<-2$ & $1.48(0.41$ to 2.56$)$ & 0.007 \\
Age in months & $0.21(0.14$ to 0.28$)$ & $<0.001$ \\
\hline Model: adjusted $r^{2}=0.46, F=76.6, p>F<0.001$. & & \\
Hb, haemoglobin. & &
\end{tabular}

during follow-up (days 30 and 90) was not associated with final $\mathrm{Hb}$ (tables 4 and 5), in children with high qPCR (quantitative PCR parasite count) parasite count $(>500 / \mu \mathrm{L}, \mathrm{N}=3)$ at day $30, \mathrm{Hb}$ at day 30 was significantly lower compared to children with 1-2 parasites/ $\mu \mathrm{L}$ or none at day 30 (82.3 vs $102.8 \mathrm{~g} / \mathrm{L} ; \mathrm{p}=0.01)$. Similarly, the mean $\mathrm{Hb}$ change was also reduced in those with persistent high parasitaemia at day $30(-5.0$ vs $8.5 \mathrm{~g} / \mathrm{L}$; mean $\mathrm{Hb}$ difference $(95 \%$ CI $) \quad 13.5 \mathrm{~g} / \mathrm{L} \quad(-0.3$ to 27.3$)$; $\mathrm{p}=0.06)$. Bone marrow response as assessed by the percentage of reticulocytes peaked at day 15 (4.5\% in both treatment groups combined), compared to similar levels of around $2 \%$ at all other measured time points (days 3, 7, 15 and 90). There was no evidence of an effect of either the postmalaria treatment group (figure 4) or of the initial antimalarial therapy (data not shown) on bone marrow response. Urinary neopterin, a product of activated macrophages and therefore a marker of a pro-inflammatory immune status, was strongly associated with qPCR parasite count at day 30 (regression coefficient: 0.2 (95\% CI 0.08 to 0.33$), \mathrm{p}=0.003$; adjusted $\mathrm{R}^{2}=0.4091$ ) and was also associated with $\mathrm{Hb}$ at day 30 . There was no evidence of an effect of the postmalaria treatment group (figure 5) or of the initial antimalarial treatment on neopterin concentrations (see online supplementary figure S3).

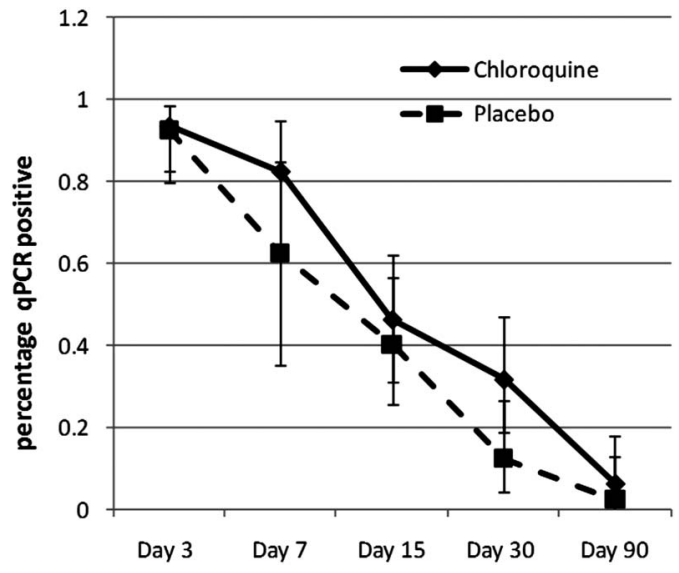

Figure 3 Percentage of participants with submicroscopic malaria (qPCR) over time by postmalarial treatment group.

\section{DISCUSSION}

Chloroquine is used in the treatment of several diseases in addition to its antimalarial use. ${ }^{28} 29$ However, we believe this to be the first study to assess its potential role in the management of postmalarial anaemia. We hypothesised that since malarial anaemia is essentially a systemic inflammatory response to infection, ${ }^{12}$ chloroquine, by suppressing plasmodium-induced inflammation, could enhance erythropoietic recovery. We tested this hypothesis by comparing two groups of childrenone group receiving weekly chloroquine and the other group weekly placebo after complete clearance of peripheral parasitaemia as determined by microscopy. The primary outcome measure was the recovery in $\mathrm{Hb}$ at days 30 and 90 compared to the day $3 \mathrm{Hb}$ levels. Our results showed no significant difference in the $\mathrm{Hb}$ change between the two groups. The data were interrogated further by a random effects model modelling data at days 30 and 90 simultaneously while adjusting for $\mathrm{Hb}$ at day 3 and taking into account random measurement errors and missing data. This confirmed that weekly chloroquine at $5 \mathrm{mg} / \mathrm{kg}$ body weight did not improve $\mathrm{Hb}$ recovery after uncomplicated clinical malaria. There was a suggestion that weekly CQ may have increased the rate of recovery in $\mathrm{Hb}$ change at day 15 accompanied by non-significantly increased reticulocyte percentage.

A limitation of the study was the need to recruit participants over two malaria transmission seasons. In 2008, there was a reduced malaria transmission rate, and thus there were fewer cases available for recruitment and also an apparent effect on the severity of malaria in terms of parasite densities at first detection and effect on

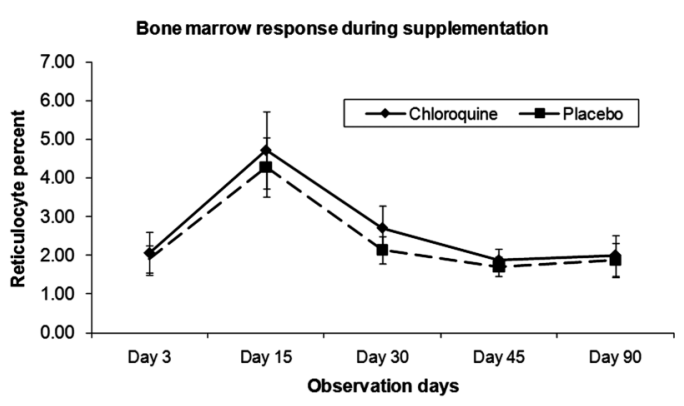

Figure 4 Reticulocyte percentage as an indicator of bone marrow response by treatment group for both years combined. 


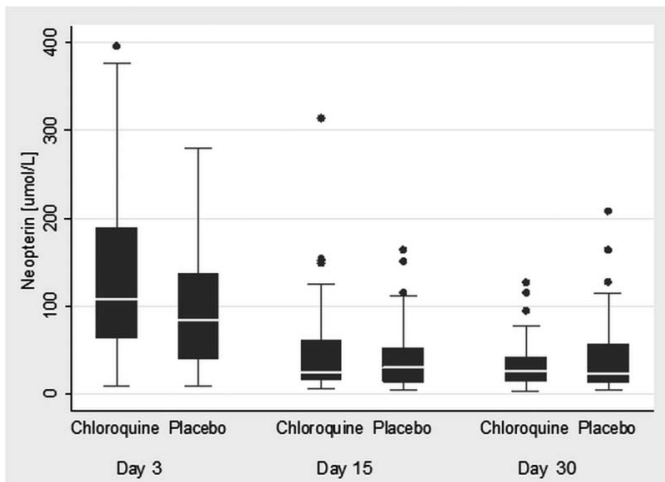

Figure 5 Urinary neopterin by postmalarial treatment group.

haemoglobin concentrations, with a significantly higher baseline $\mathrm{Hb}$ in 2008. However, the change in design that was necessitated in 2008 did not change the primary outcome analysis. Furthermore, CIs of the observed effect size in the final multivariable analysis do not include the effect size included in the sample size calculation, and thus our observation is unlikely to be due to a type II error as a result of lack of power to detect a true effect of this magnitude.

The change in design in 2008 to all children receiving ACT antimalarial treatment did not affect the analysis of the primary outcome. The change did, however, limit our ability to determine the effect of initial antimalaria therapy on haemoglobin response in the placebo group. An effect of antimalarial treatment $\mathrm{CQ}$ on $\mathrm{Hb}$ when in the context of adequate parasite clearance, as was observed, might have been expected to occur in the early period of recovery.

The recommended anti-inflammatory dose of $\mathrm{CQ}$ is 3-4 mg/ kg/day. However, owing to a lack of safety data for this condition and considering the potential adverse effects, particularly retinal damage, we used the lower prophylactic dose of $5 \mathrm{mg} / \mathrm{kg} /$ week. Using this low dose might have contributed to our inability to detect an effect of CQ. Drenou et $a l^{30}$ reported the successful long-term control of sideroblastic anaemia (a condition of reduced incorporation of iron into haem in the presence of sufficient or high iron) in an adult patient using chlrorquine but at $100 \mathrm{mg} /$ day.

We had hypothesised that chloroquine will impede macrophageal iron sequestration during the acute clinical episode and improve iron availability for the process of erythropoiesis. Urinary excretion of neopterin is markedly increased in individuals infected with malaria, and corresponds to the concurrent activation of $\mathrm{T}$ cells and macrophages. ${ }^{31-33}$ We measured the urinary neopterin at days 3, 15 and 30 and expected to see a more rapid decline in the neopterin level in the weekly chloroquine group than in the weekly placebo group, but no difference was observed between the treatment groups. It is possible that a difference may have occurred at an intermediate time point between days 3 and 15, when the levels had already almost normalised. Indeed, Brown $e t a l^{33}$ showed that in a group of volunteers experimentally infected with plasmodium parasites, the timing of maximum neopterin excretion coincided with the treatment and elimination of parasitaemia. In our study, this would coincide with study days 0-3. In future, it might be more informative to carry out more measurements during the first week of malaria diagnosis.

The participants were followed up for 90 days because the reports had suggested that $\mathrm{Hb}$ continues to fall even after successful malaria treatment primarily due to continuing inflammation. ${ }^{34}$ In this study, we found no evidence of persistent inflammation, except in the few cases with remaining parasitaemia at day 30 , which also had decreased absolute $\mathrm{Hb}$ and $\mathrm{Hb}$ change at day 30 compared with children with no parasitaemia or parasite counts of $1-2 / \mu \mathrm{L}$.

Our observation that children with higher $\mathrm{Hb}$ at diagnosis had greater $\mathrm{Hb}$ recovery is potentially paradoxical as one would have expected the reverse through statistical regression to the mean and a greater 'catch up' in children with a lower starting $\mathrm{Hb}$ at day 3 . However, as the majority of iron used for $\mathrm{Hb}$ recovery is recycled, ${ }^{24}$ those with higher $\mathrm{Hb}$ at day 0 have more iron available for recovery. Our non-provision of exogenous iron during the study may have restricted the ability of the more anaemic children to respond appropriately, as suggested by our observation of reduced $\mathrm{Hb}$ recovery in children with apparent iron deficiency at day 0. An earlier study in Gambian children showed improved haematological recovery in children treated for malaria and cosupplemented with iron compared to placebo. ${ }^{36}$ However, we have also shown in Gambian children reduced iron absorption from supplementation in postmalarial anaemia, while also experiencing greater $\mathrm{Hb}$ recovery compared to supplemented children with irondeficiency anaemia, thus causing us to question the efficacy of iron supplementation given within 2 weeks of antimalarial treatment. ${ }^{24}$ Without the inclusion of a further randomised treatment arm of postmalarial treatment iron supplementation, it is not possible to make further recommendations concerning the recent argument for the provision of iron supplementation to children in malaria endemic areas with regular malaria surveillance and treatment facilities. ${ }^{37}$

Our finding that stunted children recovered $\mathrm{Hb}$ poorly is consistent with an earlier report of stunting being a determinant of the severity of malaria-associated anaemia. ${ }^{38}$ In our population, this effect was independent of iron status.

Age has been shown to be strongly associated with risk of severe anaemia in children with acute malaria, the risk decreasing with increasing age. ${ }^{39}$ In addition, we have shown in this study that older children recover better from anaemia after an acute malaria episode than younger children. Paying special attention to younger children with malarial anaemia could reduce malariarelated mortality in children. 
In conclusion, weekly chloroquine administered for 3 months to children with mild malarial anaemia at a dose of $5 \mathrm{mg} / \mathrm{kg} /$ week resulted in no improvement in erythropoietic recovery compared to placebo. Older children and children with higher initial $\mathrm{Hb}$, less stunting at diagnosis and without iron deficiency had better $\mathrm{Hb}$ recovery postmalaria. Similar to other reports in the literature, urinary neopterin was elevated during acute malaria. The role of iron deficiency in erythropoietic recovery postmalaria noted in this study suggests areas for further detailed investigation of the clinical value in administering both antimalarial and iron supplementation to children with malarial anaemia residing in areas of high iron deficiency and malaria burden.

\section{Author affiliations}

${ }^{1}$ Medical Research Council (MRC) International Nutrition Group, London School of Hygiene \& Tropical Medicine, London, UK

${ }^{2}$ Muhimbili Wellcome Programme, Muhimbili University of Health \& Allied Sciences, Dar es Salaam, Tanzania

${ }^{3}$ MRC Keneba, Keneba, The Gambia

${ }^{4}$ Department of Paediatrics, Royal Hospital for Sick Children, Glasgow, UK

Acknowledgements The authors wish to acknowledge the contribution of all the members of the field team-Kabiru Ceesay, Edrisa Sinjanka, Lamin Ceesay, Lamin Sowe, Kalilu Sanneh, Bakary Sarr, Muhammed Sidibeh, Yahya Sanyang;the data staff, especially Jahid Hassan and Musa Jarjou; the laboratory staff, especially Musa Colley, Karamo Camara, Mustapha Bittaye and Ebrimah Danso; all the drivers of the MRC Keneba, Ms Cheryl Eniyou 0, Mrs Fatou Noho and Ms Jankey Jagne; all the village assistants; and the study subjects and their parents.

Contributors CVN, CPD and AMP conceived and designed the study with input from SEC. CVN and SEM were responsible for the study conduct and data acquisition. CVN, SEC and AJF were responsible for the statistical analysis and interpretation. SEC and CVN drafted the paper and circulated it to all authors for critical revision. All authors read and approved the final manuscript.

Funding This study was supported by the UK Medical Research Council (MC-A760-5QX00).

\section{Competing interests None.}

Ethics approval The Scientific Coordinating Committee of the Medical Research Council (UK), The Gambia, and the MRC/Gambia Government Joint Ethical Committee approved the study (SCC 1076).

Provenance and peer review Not commissioned; externally peer reviewed.

Data sharing statement The original data set is available on request to the corresponding author and permission is dependent on the nature and purpose of intended use.

\section{REFERENCES}

1. World Health Organization. Severe falciparum malaria. World Health Organization, Communicable Diseases Cluster. Trans $R$ Soc Trop Med Hyg 2000;94(Suppl 1):S1-90.

2. Murphy SC, Breman JG. Gaps in the childhood malaria burden in Africa: cerebral malaria, neurological sequelae, anemia, respiratory distress, hypoglycemia, and complications of pregnancy. Am J Trop Med Hyg 2001;64(1-2 Suppl):57-67.

3. Brabin BJ, Premji Z, Verhoeff F. An analysis of anemia and child mortality. J Nutr 2001;131(2S-2):636S-45S; discussion 46S-8S.

4. Bojang KA, Palmer A, Boele van Hensbroek M, et al. Management of severe malarial anaemia in Gambian children. Trans $R$ Soc Trop Med Hyg 1997;91:557-61.

5. Mulenga $\mathrm{M}$, Malunga $\mathrm{F}$, Bennett $\mathrm{S}$, et al. A randomised, double-blind, placebo-controlled trial of atovaquone-proguanil vs. sulphadoxine-pyrimethamine in the treatment of malarial anaemia in Zambian children. Trop Med Int Health 2006;11:1643-52.

6. Phiri KS, Calis JC, Faragher B, et al. Long term outcome of severe anaemia in Malawian children. PLOS ONE 2008;3:e2903.

7. Lackritz EM. Prevention of HIV transmission by blood transfusion in the developing world: achievements and continuing challenges. AIDS 1998;12(Suppl A):S81-6.

8. NaNA. Nationwide survey on the prevalence of Vitamin $A$ and Iron deficiency in women and children in The Gambia. National Nutrition Survey. Banjul: National Nutrition Agency/Medical Research Council, 2001.

9. Abdalla SH. Iron and folate status in Gambian children with malaria. Ann Trop Paediatr 1990;10:265-72.

10. Bojang KA, Van Hensbroek MB, Palmer A, et al. Predictors of mortality in Gambian children with severe malaria anaemia. Ann Trop Paediatr 1997;17:355-9.

11. Brewster DR, Greenwood BM. Seasonal variation of paediatric diseases in The Gambia, West Africa. Ann Trop Paediatr 1993;13:133-46.

12. Nweneka CV, Doherty CP, Cox S, et al. Iron delocalisation in the pathogenesis of malarial anaemia. Trans $R$ Soc Trop Med Hyg 2010;104:175-84.

13. Evans KJ, Hansen DS, van Rooijen N, et al. Severe malarial anemia of low parasite burden in rodent models results from accelerated clearance of uninfected erythrocytes. Blood 2006;107:1192-9.

14. Price RN, Simpson JA, Nosten F, et al. Factors contributing to anemia after uncomplicated falciparum malaria. Am J Trop Med Hyg 2001;65:614-22.

15. Ganz T. Molecular pathogenesis of anemia of chronic disease. Pediatr Blood Cancer 2006;46:554-7.

16. Ganz T. Hepcidin and its role in regulating systemic iron metabolism. Hematology Am Soc Hematol Educ Program 2006:29-35, 507.

17. Prentice AM, Doherty CP, Abrams SA, et al. Hepcidin is the major predictor of erythrocyte iron incorporation in anemic African children. Blood 2012;119:1922-8.

18. Nemeth E, Rivera S, Gabayan V, et al. IL-6 mediates hypoferremia of inflammation by inducing the synthesis of the iron regulatory hormone hepcidin. J Clin Invest 2004;113:1271-6.

19. Lee $\mathrm{P}$, Peng $\mathrm{H}$, Gelbart $\mathrm{T}$, et al. Regulation of hepcidin transcription by interleukin-1 and interleukin-6. Proc Natl Acad Sci USA 2005;102:1906-10.

20. Atkinson SH, Rockett KA, Morgan G, et al. Tumor necrosis factor SNP haplotypes are associated with iron deficiency anemia in West African children. Blood 2008;112:4276-83.

21. Legssyer R, Ward RJ, Crichton RR, et al. Effect of chronic chloroquine administration on iron loading in the liver and reticuloendothelial system and on oxidative responses by the alveolar macrophages. Biochem Pharmacol 1999;57:907-11.

22. Ghigo D, Aldieri E, Todde R, et al. Chloroquine stimulates nitric oxide synthesis in murine, porcine, and human endothelial cells. J Clin Invest 1998;102:595-605.

23. Atkinson SH, Rockett K, Sirugo G, et al. Seasonal childhood anaemia in West Africa is associated with the haptoglobin 2-2 genotype. PLoS Med 2006;3:e172.

24. Doherty CP, Cox SE, Fulford AJ, et al. Iron incorporation and post-malaria anaemia. PLOS ONE 2008;3:e2133.

25. Worwood M. The laboratory assessment of iron status-an update. Clin Chim Acta 1997;259:3-23.

26. Hershko C, Bar-Or D, Gaziel Y, et al. Diagnosis of iron deficiency anemia in a rural population of children. Relative usefulness of serum ferritin, red cell protoporphyrin, red cell indices, and transferrin saturation determinations. Am J Clin Nutr 1981;34:1600-10.

27. Nwakanma DC, Gomez-Escobar N, Walther M, et al. Quantitative detection of Plasmodium falciparum DNA in saliva, blood, and urine. $J$ Infect Dis 2009;199:1567-74.

28. Cooper RG, Magwere T. Chloroquine: novel uses \& manifestations. Indian J Med Res 2008;127:305-16.

29. Wallace DJ. The use of chloroquine and hydroxychloroquine for non-infectious conditions other than rheumatoid arthritis or lupus: a critical review. Lupus 1996;5(Suppl 1):S59-64.

30. Drenou B, Guyader D, Turlin B, et al. Treatment of sideroblastic anemia with chloroquine. N Engl J Med 1995;332:614.

31. Reibnegger G, Boonpucknavig V, Fuchs $\mathrm{D}$, et al. Urinary neopterin is elevated in patients with malaria. Trans $R$ Soc Trop Med Hyg 1984;78:545-6.

32. Fuchs D, Hausen A, Reibnegger G, et al. Neopterin as a marker for activated cell-mediated immunity: application in HIV infection. Immunol Today 1988;9:150-5. 
33. Brown AE, Herrington DA, Webster HK, et al. Urinary neopterin in volunteers experimentally infected with Plasmodium falciparum.

Trans R Soc Trop Med Hyg 1992;86:134-6.

34. Dondorp AM, Nyanoti M, Kager PA, et al. The role of reduced red cell deformability in the pathogenesis of severe falciparum malaria and its restoration by blood transfusion. Trans $R$ Soc Trop Med Hyg 2002;96:282-6.

35. Biemba G, Gordeuk VR, Thuma PE, et al. Prolonged macrophage activation and persistent anaemia in children with complicated malaria. Trop Med Int Health 1998;3:60-5.

36. van Hensbroek MB, Morris-Jones S, Meisner S, et al. Iron, but not folic acid, combined with effective antimalarial therapy promotes haematological recovery in African children after acute falciparum malaria. Trans R Soc Trop Med Hyg 1995;89:672-6.

37. Ojukwu JU, Okebe JU, Yahav D, et al. Oral iron supplementation for preventing or treating anaemia among children in malaria-endemic areas. Cochrane Database Syst Rev 2009(3):CD006589.

38. Verhoef $\mathrm{H}$, West $\mathrm{CE}$, Veenemans $\mathrm{J}$, et al. Stunting may determine the severity of malaria-associated anemia in African children. Pediatrics 2002;110:e48.

39. Reyburn H, Mbatia R, Drakeley C, et al. Association of transmission intensity and age with clinical manifestations and case fatality of severe Plasmodium falciparum malaria. JAMA 2005:293:1461-70 